\title{
Identificación de las principales plagas que afectan la pitahaya (Hylocereus undatus Britt and Rose) en Carazo, Nicaragua, 2018
}

\author{
Identification of main pests that affect pitahaya in Carazo, Nicaragua, 2018
}

\author{
Edgardo Jiménez Martinez ${ }^{4}$ \\ Cristiam Jesús López Avilés ${ }^{5}$ \\ Dereck Beirut Espinoza Borges ${ }^{6}$
}

\section{Resumen}

La pitahaya (Hylocereus undatus, Britt and Rose) es una planta que pertenece a la familia cactáceae, en Nicaragua esta se siembra principalmente en los departamentos de Masaya y Carazo, se reportan unas 700 hectáreas de pitahaya, indicando que Nicaragua es el principal productor en Centroamérica con una producción nacional de más 6160 toneladas, de las cuales se exportan más del 50\%. En Nicaragua hay poca información con respecto a las principales plagas que afectan el cultivo de pitahaya y no hay reportes exhaustivos de plagas primarias, por tal razón se estableció un estudio con el objetivo de identificar las principales plagas presentes en el cultivo de la pitahaya en el municipio de La Concha, Masaya, la investigación se realizó entre los meses de julio a noviembre del 2017, los genotipos de pitahaya evaluados fueron Orejona, Rosa, Lisa, Chocoya, Sabaneña, y Espinuda. El diseño del estudio utilizado en la investigación cualitativa no experimental que se fundamenta en el método descriptivo, forma de investigación aplicada en donde no hay manipulación de variables. Se encontraron 9 órdenes y 40 familias de insectos asociados a la pitahaya, las familias más dominantes fueron en la Finca Palo Solo Phycitidae y Noctuidae y en la Finca Panamá fue Chrysopidae y Hesperiidae.

Palabras clave: Insectos; cactáceae; abundancia; riqueza.

\section{Summary}

Pitahaya (Hylocereus undatus, Britt and Rose) is a plant that belongs to the cactaceae family, in Nicaragua it is sown mainly in the departments of Masaya and Carazo, about 700 hectares of pitahaya are reported, indicating that Nicaragua is the main producer in Central America with a national production of more than 6,160 tons, of which more than $50 \%$ are exported. In Nicaragua there is little information regarding

\footnotetext{
$4 \mathrm{PhD}$ en Entomología, docente investigador, Director de Investigación, Extensión y Posgrado de la Universidad Nacional Agraria, Nicaragua. Correo: edgardo.jimenez@ci.una.edu.ni; No. ORCID: https://orcid.org/oooo-0003-1086-7380

5 Ingeniero Agrónomo. Universidad Nacional Agraria, Nicaragua.

6 Ingeniero Agrónomo. Universidad Nacional Agraria, Nicaragua.
}

Recibido: 26/03/2020 Aprobado: 28/05/2020 
the main pests that affect the pitahaya crop and there are no exhaustive reports of primary pests, for this reason a study was established with the aim of identifying the main pests present in the pitahaya crop in La Concha municipality, Masaya, the research was carried out between the months of July to November 2017, the pitahaya genotypes evaluated were Orejona, Rosa, Lisa, Chocoya, Sabaneña, and Espinuda. The study design used in non-experimental qualitative research that is based on the descriptive method, a form of applied research where there is no manipulation of variables. 9 orders and 40 families of insects were found associated with pitahaya, the most dominant families were in Palo Solo Farm Phycitidae and Noctuidae and in Panamá Farm it was Chrysopidae and Hesperiidae.

Keywords: Insects; cactaceae; abundance; richness.

\section{Introducción}

La pitahaya (Hylocereus undatus, Britt and Rose) es una planta epifita y originaria de América, pertenece a la familia de las Cactáceas, es una planta perenne, que crece de forma silvestre sobre árboles vivos, troncos secos, piedras y muros, su arquitectura no le impide sostenerse a sí misma (Avelares et al., 1996). El cultivo de la pitahaya está mayormente distribuido en el Pacífico del país, principalmente en León, Chinandega, Masaya, Carazo, Granada, Rivas; en la zona norte en Estelí, Boaco y Chontales (INTA, 2002). (Se recomienda no iniciar primer párrafo citando a otros, sino a lo qu ella sabe el autor del artículo).

El propósito de esta investigación es conocer qué tipo de insectos están asociados al cultivo de pitahaya y la distribución temporal de estos insectos ya que, los productores conocen muy poco de las especies que se encuentran en su entorno y por esta razón se les dificulta diferenciar cual realmente es la plaga. También se toma en cuenta la necesidad de caracterizar los genotipos con los que actualmente se trabaja en Nicaragua y diferenciar entre distintas características, cual resulta ser el más factible al momento de establecer una plantación.

A nivel comercial hay dos especies de pitahaya, la especie (Hylocereus triangularis Haw), conocidas como pitahaya amarilla que se siembra en Colombia; y la especie pitahaya, cuya pulpa es de color rojo intenso hasta morado, cultivada en Nicaragua, el sur de México, Guatemala y el Salvador. En cada país productor de pitahaya existen varias especies e incluso en la misma especie dominante hay gran polimorfismo. Son cuatro grupos de pitahayas, de los cuales tres son los de mayor importancia comercial: 1) pitahaya amarilla, 2) pitahaya roja de pulpa blanca y 3 ) pitahaya roja de pulpa roja (Becerra, 1986).

La demanda semanal de países consumidores como Estados Unidos (50 ton), Europa (30 ton), Japón (20 ton) también se ha visto el incremento dada la cantidad 
de inmigrantes provenientes de países productores, quienes exigen frutas tropicales de su país de origen (Rosses, 1999). Además, se tienen grandes perspectivas de exportaciones de frutas a los Estados Unidos, ya que la pitahaya no es hospedera de la mosca del mediterráneo (Ceratitis Capitata, Wied), lo que fue confirmado por técnicos especialistas de la Secretaría de la Agricultura de los Estados Unidos (USDA) y el Ministerio de Agricultura (MAG) de Nicaragua (INTA, 2002).

En Nicaragua la pitahaya tiene una producción escalonada desde mayo a noviembre; dependiendo de los meses de lluvias y los clones, las cosechas se pueden extender hasta el mes de octubre. Agosto y septiembre son los de mayor producción en el Pacífico de Nicaragua, la cosecha oportuna y bien realizada, así como el adecuado manejo postcosecha, garantizan una mejor calidad del fruto a comercializar y un aumento de la vida comercial del mismo (MIFIC, 2001).

El cultivo de pitahaya tiene un crecimiento acelerado en nuestro país con respecto a las áreas cultivadas y a las exportaciones de la fruta; sin embargo, en la parte de producción existen todavía muchos desconocimiento en cuanto al entendimiento de todos los aspectos que involucra el cultivo, motivo por el cual se realizó esta investigación para conocer algunos aspectos como: la caracterización de los genotipos con los que actualmente trabajan los productores en la zona que más produce esta fruta, los insectos y patógeno asociados a este tipo de cultivo.

En el estudio realizado se encontraron 9 órdenes y 40 familias de insectos asociados a la pitahaya, las familias más dominantes en la Finca Palo Solo fueron Phycitidae y Noctuidae y en la Finca Panamá fueron Chrysopidae y Hesperiidae.

La mayor abundancia, riqueza y diversidad de insectos asociados al cultivo de la pitahaya fue encontrada en la finca Palo Solo y los mayores picos poblacionales en su distribución temporal fueron encontrados en las familias Noctuidae y Chrysopidae. La mayor captura de insectos fue registrada en la trampa de caída libre en la finca Palo solo

Se recomienda establecer los genotipos Orejona y Sabaneña por sus características morfológicas y la tolerancia a enfermedades.

Se recomienda asociar el cultivo de pitahaya con otros cultivos alimenticios para que la diversidad insectil aumente.

\section{Materiales y métodos}

\section{Ubicación del lugar de estudio}

El estudio se realizó entre los meses de julio a noviembre del 2017, en la empresa Burke Agro S.A que se encarga en el procesamiento y exportación de frutas como 
pitahaya, la empresa está ubicada en el departamento de Carazo, municipio San Marco, con una posición geográfica $11^{\circ} 54^{\prime} 03.1^{\prime N} 86^{\circ} 12^{\prime} 06.9^{\prime \prime ~ W . ~ E l ~ m u n i c i p i o ~ d e ~ S a n ~ M a r c o ~}$ cuenta con una extensión territorial de 118.11 kilómetros cuadrados, con altitud sobre el nivel del mar de (552.40 metros). Según INETER (2008), las precipitaciones promedio anuales en el departamento de Carazo son de $1434.5 \mathrm{~mm}$ y una temperatura de $26.4^{\circ} \mathrm{C}$.

La finca "Palo solo" está ubicada en la comunidad de San Ignacio, propiedad del Sr. Julio con una parcela de $7026 \mathrm{~m}^{2}$. La finca "Panamá" también está ubicada en la comunidad de San Ignacio propiedad del Sr. Martín con una parcela de $7236 \mathrm{~m}^{2}$.

En el diseño del estudio utilizado en la investigación cualitativa no experimental que se fundamenta en el método descriptivo, forma de investigación aplicada en donde no hay manipulación de variables (UNA, 2008). Consistió en la comparación de 2 fincas de producción de pitahaya ubicadas en el Municipio de La Concepción, Comunidad de San Ignacio, cabe mencionar que en cada finca se colocaron 6 trampas, de las cuales 3 de ellas eran trampas de caída libre y 3 trampas de galón con melaza.

\section{Muestreo y descripción de los principales insectos asociados al cultivo de pitahaya}

El muestreo o colecta de insectos se realizó cada 15 días, en dos fincas del departamento de Masaya. El muestreo se realizó utilizando dos métodos. El primer método consistió, en la captura de insectos rastreros con trampas de caída libre (Pitfall traps) el segundo método de colecta consistió en la captura de insectos voladores y caminadores, utilizando trampas de recipientes plásticos galones de color blanco Anexo, 7, para la captura se le agregaba al recipiente una mezcla de Xedex ${ }^{\circledR}$ y melaza, ácido bórico, jugo de vaina de pitahaya y jugo del fruto de la pitahaya. La colecta de los insectos hizo en viales entomológicos, las cuales fueron rotulados con la fecha y el sitio de colecta, posteriormente estos insectos fueron llevados al laboratorio de entomología de la UNA para ser montados e identificados.

\section{Descripción del primer método de captura de insectos}

Para la captura y colecta de insectos rastreros y algunos voladores se utilizaron trampas de caída libre (Pitfall-traps), este consistió en colocar panas plásticas de color azul de $30 \mathrm{~cm}$ de diámetro y $15 \mathrm{~cm}$ de profundidad al ras del suelo con capacidad de dos litros de agua, a estas se le agregó 5 gramos de detergente del tipo Xedex ${ }^{\circledR}$, en dos litros de agua, $100 \mathrm{ml}$ de jugo de vaina de pitahaya, 5 gramos de ácido bórico y 10 $\mathrm{cm}^{3}$ de melaza, la frecuencia de colecta hizo cada 15 días. En cada fecha de muestreo se cambió la solución antes descrita (Montano y Bustamante, 2016). 


\section{Descripción del segundo método de muestreo}

Se utilizó trampas de galones plásticos de color blanco con capacidad de 3.78 litros de agua y se agregó 5 gramos de detergente $\mathrm{Xedex}^{\circledR}$, ácido bórico y jugo de pitahaya, como un atrayente para los insectos voladores y dos litros de agua, los galones fueron cortados por los dos costados arriba de la mitad y colgados con alambre verticalmente a una altura de $1.5 \mathrm{~m}$. La frecuencia de colecta fue cada 15 días, en cada fecha de muestreo se cambió la solución antes descrita.

\section{Muestras e identificación de insectos a nivel de laboratorio}

El procesamiento de las muestras de insectos en el laboratorio consistió inicialmente en sacar el espécimen de los vasos colectores por fecha y por finca, estos se vaciaron individualmente sobre papel toalla, posteriormente con la ayuda de pinceles finos. Se realizó la separación de los insectos capturados y para el montaje de los especímenes se utilizó alfileres entomológicos (MORPHO de $4 \mathrm{~cm}$ de longitud), para la identificación de los especímenes se utilizó estereoscopios (CARL ZEISS, modelo 475002 de 4x, 6.3x y 2.5x).

La identificación se hizo hasta un nivel de familia, esta se realizó en el laboratorio de entomología de la UNA; para la identificación de familias se utilizaron claves taxonómicas dicotómicas propuestas por Nunes y Dávila (2004). Una vez identificados los insectos a nivel de familia, se procedió a la identificación hasta el taxón de género y especie.

También se consultaron otras literaturas, taxonomía de las principales familias y subfamilias de insectos de interés agrícolas en Nicaragua (Andrews y Caballero, 1989), texto básico: entomología (Jiménez, 2009), insectos de Nicaragua: catálogo de los insectos y artrópodos terrestres de Nicaragua (Maes, 1998) y el texto de entomología sistemática (Sáenz y de la Llana, 1990).

\section{Variables evaluadas en el estudio}

Principales órdenes, familias, géneros, especies, nombre común y hábitos alimenticios

\section{Abundancia total de insectos encontrados por finca}

Esta variable se comenzó a tomar desde la fecha 15 de julio, cada 15 días hasta el 12 de noviembre; Se realizó un conteo de todos los insectos colectados en las trampas Pitfall traps y trampa de galón. 


\section{Abundancia de insectos de los principales órdenes encontrados por finca}

Se tomaron en cuenta los principales órdenes, Díptera, Hymenóptera, Coleópteray Lepidóptera, Hemíptera, se contaron el total de insectos en todas las fechas de colecta por tipo de trampa.

\section{Comparación de abundancia de insectos por familia encontrados por finca}

Se hizo un conteo de la abundancia de insectos de las diferentes familias encontradas en todas las fechas de colectas, para determinar cuál era la finca con mayor número de familias.

\section{Riqueza total de familias de insectos por finca}

Se hizo un conteo por familia encontrado en todas las fechas de colecta de insectos en la finca, para determinar cuál era la finca con mayor riqueza en cuanto a familia se refiere.

\section{Distribución temporal de las principales familias encontradas por finca}

Se comparó la distribución temporal de las principales familias de insectos Noctuidae, Chrysopidae, Phycitidae por fecha de colecta, donde se hizo la sumatoria del número de insectos encontrados por familia en cada fecha de muestreo.

\section{Análisis de los datos}

Una vez colectados los insectos en el campo, estos fueron ordenados por variables de familias de insectos por finca en una tabla de datos en Excel, luego cada variable será comparada entre fincas, utilizando un análisis de comparación de t de student, usando el programa de Infostat (versión, 2016). El nivel de significancia usado en el análisis fue de $(\mathrm{p}=0.05)$.

\section{Resultados y discusión}

\section{Principales órdenes, familias, géneros, especies, nombre común y hábitos alimenticios de insectos encontrados en el cultivo de la pitahaya}

Los principales órdenes de insectos recolectados en este estudio son presentados a continuación. Es importante mencionar que son pocos los estudios de pitahaya relacionados con este tipo de estudio a nivel nacional y regional. En el Cuadro 1, se presentan los principales órdenes, familias, géneros, especies, nombre común y habito alimenticio de insectos encontrados en las fincas evaluadas Palo Solo y Panamá en el período comprendido entre julio a noviembre del 2017. Dentro de los individuos de 
insectos podemos encontrar algunos órdenes de insectos como Coleóptera, Dermáptera, Lepidóptero, Orthóptera, Díptera, Hymenóptera, Neuróptera, Hemíptera, Blattodea y con hábitos alimenticios: depredador, coprófago, fitófago, polífago, nectarívoro y omnívoro.

Cuadro No. 1: Órdenes, familias, géneros, especies, nombre común y hábitos alimenticios de insectos encontrados en el cultivo de la pitahaya

\begin{tabular}{|c|c|c|c|c|c|}
\hline Orden & Familia & Género & Especie & Nombre común & Hábito alimenticio \\
\hline \multirow{27}{*}{ Coleóptera } & Curculionidae & Catophagus & Sp & Picudo & Fitófago \\
\hline & Carabidae & Scarites & Sp & Escarabajo & Depredador \\
\hline & Scarabidae & Deltochilum & Sp & Escarabajo Pelotero & Coprófago \\
\hline & Curculionidae & Antonomus & grandis & Picudo & Fitófago \\
\hline & Curculionidae & Antonomus & grandis & Picudo & Fitófago \\
\hline & Carabidae & Calleida & $\mathrm{sp}$ & Escarabajo & Coprófago \\
\hline & Scarabidae & Anomala & $s p$ & Chocorrón & Fitófago \\
\hline & Cerambycidae & Cylene & $\mathrm{sp}$ & Escarabajo & Coprófago \\
\hline & Scarabidae & Canthon & $\mathrm{sp}$ & Escarabajo Pelotero & Coprófago \\
\hline & Tenebrionidae & Glytasida & $s p$ & & Fitófago \\
\hline & Elateridae & Conoderus & $\mathrm{sp}$ & Insecto Clic & Fitófago \\
\hline & Scarabidae & Cotinus & mutabilis & Chocorrón & Polífago \\
\hline & Scarabidae & Phylophaga & $\mathrm{sp}$ & Gallina Ciega & Fitófago \\
\hline & Lycidae & Calopteron & $\mathrm{sp}$ & Mosquita & Fitófago \\
\hline & Passalidae & Passalus & $\mathrm{sp}$ & Escarabajo & Fitófago \\
\hline & Scarabidae & Euphoria & $\mathrm{sp}$ & Gallina Ciega & Fitófago \\
\hline & Chrysomelidae & Cerotoma & $\mathrm{sp}$ & & Fitófago \\
\hline & Carabidae & Colosoma & soyi & & Fitófago \\
\hline & Histeridae & Hololepta & $\mathrm{sp}$ & Escarabajo & Fitófago \\
\hline & Staphylidae & Paederus & $\mathrm{sp}$ & Culebrilla & Depredador \\
\hline & Elateridae & Pyrophorus & $\mathrm{sp}$ & Escarabajo & Polífago \\
\hline & Cerambycidae & Lagocheirus & $\mathrm{sp}$ & & Fitófago \\
\hline & Coccinelidae & Cyclonida & sanguínea & Mariquita & Depredador \\
\hline & Carabidae & Calleida & $\mathrm{sp}$ & & Fitófago \\
\hline & Elateridae & Aeolus & $\mathrm{sp}$ & Gusano Alambre & Fitófago \\
\hline & Elateridae & Conoderus & $\mathrm{sp}$ & Escarabajo & Fitófago \\
\hline & Tenebrionidae & Celenophors & $\mathrm{sp}$ & Escarabajo & Fitófago \\
\hline Neuróptera & Chrysopidae & Chrysoperla & sp & León de afidos & Depredador \\
\hline
\end{tabular}




\section{AGROPECUARIO}

\begin{tabular}{|c|c|c|c|c|c|}
\hline Orden & Familia & Género & Especie & Nombre común & Hábito alimenticio \\
\hline \multirow{7}{*}{ Hymenóptera } & Apidae & Apis & mellifera & Aveja & Nectarívoro \\
\hline & Vespidae & Polistes & $\mathrm{sp}$ & Cátala & Depredador \\
\hline & Sphecidae & Sceliphon & $\mathrm{sp}$ & Avispa & Depredador \\
\hline & Formicidae & Componotus & $\mathrm{sp}$ & Hormiga & Fitófago \\
\hline & Halictidae & Halictus & $\mathrm{sp}$ & & Nectarivoro \\
\hline & Formicidae & Dinoponera & $\mathrm{sp}$ & Hormiga & Fitófago \\
\hline & Vespidae & Polybia & $\mathrm{sp}$ & Avispa & Nectarívoro \\
\hline \multirow{5}{*}{ Orthóptera } & Acrididae & Orphulela & $\mathrm{sp}$ & Saltamontes & Fitófago \\
\hline & Gryllidae & Acheta & similis & Grillo & Omnívoro \\
\hline & Gryllidae & Acheta & $\mathrm{sp}$ & Grillo & Omnívoro \\
\hline & Redubidae & estadoninfal & & & Omnívoro \\
\hline & Membracidae & Cimbonia & $\mathrm{sp}$ & & Coprofago \\
\hline \multirow{8}{*}{ Hemíptera } & Membracidae & Athiante & $\mathrm{sp}$ & Chinche & Fitófago \\
\hline & Pentatomidae & Punctulatus & $\mathrm{sp}$ & Chinche & Fitófago \\
\hline & Cicadellidae & Oricometopia & $\mathrm{sp}$ & Chicharra & Fitófago \\
\hline & Galastocoridae & Galastocris & $\mathrm{sp}$ & & Fitófago \\
\hline & Membracidae & Spyssistilus & $\mathrm{sp}$ & Chicharra & Fitófago \\
\hline & Cercopidae & Aenolamia & postica & Salivazo & Fitófago \\
\hline & Cercopidae & Prosapia & $\mathrm{sp}$ & Salivazo & Fitófago \\
\hline & Tipulidae & & & & Fitófago \\
\hline \multirow{6}{*}{ Díptera } & Muscudae & Musca & domestica & Mosca & Coprófago \\
\hline & Calliphoridae & Cochomia & $\mathrm{sp}$ & & Coprófago \\
\hline & Sarcophagidae & Sarcophaga & $\mathrm{sp}$ & Mosca & Coprófago \\
\hline & Techihidae & Lespecia & $\mathrm{sp}$ & Mosca & Coprófago \\
\hline & Muscidae & Musca & $\mathrm{sp}$ & Mosca & Coprófago \\
\hline & Calliphoridae & Coehliomya & $\mathrm{sp}$ & Mosca & Coprófago \\
\hline Dermáptera & Forficulidae & Darus & linearis & Tijereta & Depredador \\
\hline Blattodea & Blatellidae & Supella & $\mathrm{sp}$ & Cucaracha & Coprófago \\
\hline \multirow{4}{*}{ Lepidóptera } & Noctuidae & spodoptera & sunia & Palomilla & $\begin{array}{l}\text { Chupador tubo } \\
\text { sifón }\end{array}$ \\
\hline & Pyralidae & Diatrea & $\mathrm{sp}$ & Barredor & Nectarívoro \\
\hline & Hesperiidae & Urbanus & $\mathrm{sp}$ & Papalote & Nectarívoro \\
\hline & Noctuidae & Mocis & lepis & Palomilla & Nectarívoro \\
\hline
\end{tabular}




\section{Abundancia de órdenes insectiles encontrados en las fincas Palo Solo y Panamá en el cultivo de la pitahaya}

Los principales órdenes de insectos encontrados en el estudio fueron Díptera, Hymenóptera, Coleóptera, Lepidóptera, Hemíptera, Neuróptera, Orthóptera, Dermáptera, Blattodea (Cuadro 2). Al comparar la abundancia de estos nueve órdenes de insectos se encontró que hubo mayor cantidad de insectos por orden en la finca Palo Solo con nueve órdenes en cada una de las fincas.

Los insectos del orden Hymenóptera como por ejemplo los de la familia Apidae y Vespidae, son polinizadores, producen miel, son agentes de control natural y biológico de plagas en los cultivos. Los insectos del orden Coleóptera tienen hábitat y alimentación variables como por ejemplo Scarabidae, Chrysomelidae y Coccinellidae, pueden ser (minadores, barrenadores, trozadores, defoliadores, predadores entre otros), También son plagas de cultivos, predadores de plagas y malezas, participan en reciclaje de la materia orgánica. Lepidóptera ejemplo la familia Noctuidae y Phyralidae son larvas generalmente fitófagas (mayormente fitófago externos y pocos minadores de hojas), son predadores y parasitas. Reciben nombres como cortadores, trozadores, soldados, medidores y barrenadores. Hemíptera, ejemplo la familia Coreidae, Coccidae y Fulgoridae la mayoría son fitófagos se alimentan principalmente de la savia de las plantas, algunos son depredadores de plagas y malezas. (Sáenz y De la Llana, 1990).

Se encontró que los tres órdenes con mayor abundancia de insectos en la finca Palo Solo y Panamá, Lepidóptera (Los cuales son insectos en su mayoría nectarívoros, en su etapa larval presentan hábitos fitófagos y resultan ser plagas de los cultivos. ), los del orden Hymenóptera (son polinizadores en su mayoría como el Apis que son los que se encuentran polinizando la flor de la pitahaya), otros insectos como del orden Coleóptera (Son en su mayoría depredadores y otros con hábitos coprófagos y fitófagos debido a que la pitahaya requiere de un tutor para sostenerse muchos coleópteros lo utilizan de refugió y de alimento a la vez) por eso son los órdenes más abundantes mencionados anteriormente.

Cuadro No. 2: Abundancia de órdenes insectiles encontrados en las fincas Palo Solo y Panamá en el cultivo de pitahaya

\begin{tabular}{|l|c|c|c|}
\hline \multicolumn{1}{|c|}{ Ordenes } & \multicolumn{2}{c|}{ Número de insectos por finca } & \\
\hline & Finca Palo Solo & Finca Panamá & Total \\
\hline Neuróptera & 124 & 175 & 299 \\
\hline Hymenóptera & 193 & 183 & 376 \\
\hline Orthóptera & 65 & 78 & 143 \\
\hline Dermáptera & 134 & 94 & 228 \\
\hline Hemíptera & 117 & 75 & 192 \\
\hline
\end{tabular}




\begin{tabular}{|l|c|c|c|}
\hline \multicolumn{1}{|c|}{ Ordenes } & \multicolumn{2}{c|}{ Número de insectos por finca } & \\
\hline & Finca Palo Solo & Finca Panamá & Total \\
\hline Coleóptera & 234 & 189 & 423 \\
\hline Blattodea & 14 & 6 & 20 \\
\hline Lepidóptera & 352 & 235 & 587 \\
\hline Díptera & 92 & 78 & 170 \\
\hline Total & 1325 & 1113 & 2438 \\
\hline
\end{tabular}

\section{Comparación de la abundancia de los principales insectos de las familias encontradas en el cultivo}

En el cuadro 3 se muestra que la mayor cantidad familias de insectos en la finca Palo Solo, encontraron 36 familias y 32 familias de insectos en la finca Panamá. El total de familias encontradas en ambas fincas fue de 40.

La familia más abundante en este caso fue la Chrysopidae, encontrándose el mayor número de insectos en la finca Panamá con 175 y siendo menor en Palo Solo con 124 insectos, esta familia fue constante, presentando los valores más altos durante todas las fechas de colecta. Respecto a sus hábitos alimenticios se sabe que los insectos de esta familia son zoófago en su mayoría depredadores, que se alimentan de otras especies y en condiciones de escases se alimentan de secreciones. Estos estaban presentes en ambas fincas, probablemente fueron atraídos por la melaza, a eso se le atribuye la mayor abundancia de insectos encontrados en el cultivo de pitahaya.

La familia Noctuidae fue la que se presentó en segundo lugar con mayor número de individuos (272 insectos), encontrándose el mayor número de insectos en la finca Palo Solo con 200 insectos y en la finca Panamá se encontró 72 insectos, cabe mencionar que esta familia fue constante en todas las fechas de colecta. Respecto a sus hábitos alimenticios los insectos de esta familia algunos son nectarívoros y fitófagos en su etapa larval.

La familia Phycitidae fue la tercera más abundante, encontrándose el mayor número de insectos únicamente en la finca Palo Solo con 252 insectos, esta familia se presentó en todas las fechas de colecta. Respecto a sus hábitos alimenticios los insectos de esta familia son nectarívoros y fitófagos en la etapa larval.

Cuadro No. 3. Comparación de la abundancia de insectos de las familias encontradas en el cultivo

\begin{tabular}{|l|l|l|c|}
\hline \multicolumn{1}{|c|}{ Familias } & \multicolumn{2}{|c|}{ Espécimen capturado por finca } & Total \\
\hline & Palo Solo & Panamá & \\
\hline Phycitidae & 252 & 0 & 252 \\
\hline
\end{tabular}




\begin{tabular}{|c|c|c|c|}
\hline \multirow[t]{2}{*}{ Familias } & \multicolumn{2}{|c|}{ Espécimen capturado por finca } & \multirow[t]{2}{*}{ Total } \\
\hline & Palo Solo & Panamá & \\
\hline Noctuidae & 200 & 72 & 272 \\
\hline Forficulidae & 134 & 94 & 228 \\
\hline Chrysopidae & 124 & 175 & 299 \\
\hline Cicadellidae & 65 & 0 & 65 \\
\hline Apidae & 57 & 37 & 94 \\
\hline Membracidae & 56 & 16 & 72 \\
\hline Vespidae & 54 & 51 & 105 \\
\hline Gryllidae & 49 & 17 & 66 \\
\hline Tenebrionidae & 37 & 11 & 48 \\
\hline Fomicidae & 36 & 0 & 36 \\
\hline Calliphoridae & 35 & 27 & 62 \\
\hline Carabeidae & 35 & 25 & 60 \\
\hline Pentatomidae & 32 & 0 & 32 \\
\hline Sphecidae & 26 & 58 & 84 \\
\hline Scarabaeidae & 26 & 28 & 54 \\
\hline Sarcophagidae & 26 & 27 & 53 \\
\hline Coccinelidae & 23 & 16 & 39 \\
\hline Reduviidae & 23 & 13 & 36 \\
\hline Fulgoridae & 23 & 0 & 23 \\
\hline Halictidae & 20 & 32 & 52 \\
\hline Tipulidae & 20 & 0 & 20 \\
\hline Gelastoridae & 19 & 12 & 31 \\
\hline Acrididae & 16 & 14 & 30 \\
\hline Histeridae & 16 & 0 & 16 \\
\hline Lycidae & 15 & 18 & 33 \\
\hline Staphylinidae & 14 & 10 & 24 \\
\hline Blatteridae & 14 & 6 & 20 \\
\hline Ichneumonidae & 13 & 28 & 41 \\
\hline Passalidae & 13 & 12 & 25 \\
\hline Elateridae & 12 & 12 & 24 \\
\hline Muscidae & 11 & 37 & 48 \\
\hline Chrysomelidae & 11 & 6 & 17 \\
\hline Cerambycidae & 7 & 19 & 26 \\
\hline Curculionidae & 7 & 15 & 22 \\
\hline
\end{tabular}




\begin{tabular}{|l|l|l|l|}
\hline \multicolumn{1}{|c|}{ Familias } & \multicolumn{2}{c|}{ Espécimen capturado por finca } & \multicolumn{1}{c|}{ Total } \\
\hline & \multicolumn{1}{|c|}{ Palo Solo } & \multicolumn{1}{c|}{ Panamá } & \\
\hline Nitidulidae & 4 & 17 & 21 \\
\hline Hesperiidae & 0 & 173 & 173 \\
\hline Cercopidae & 0 & 34 & 34 \\
\hline Tachenidae & 0 & 34 & 34 \\
\hline
\end{tabular}

\section{Abundancia total de insectos encontrados en el cultivo de la pitahaya}

La abundancia total de insectos encontrados durante el período de estudio en las dos fincas de pitahaya fue de 2438 insectos, siendo la finca Palo Solo donde se encontró el mayor número de insectos durante todo el período de colecta con 1,433 en total, mientras que en la finca Panamá, se encontraron 1005 insectos en total (Figura, 1).

En un estudio realizado por Gómez (2011), reporta que encontró una abundancia total de 3825 insectos utilizando el mismo método de captura en el cultivo de marañón, en cambio en el cultivo de pitahaya con el mismo tipo de trampa, la abundancia total fue de 2438 insectos.

Téllez y Jirón (2014), encontraron una abundancia total de 6064 insectos, utilizando el mismo método de captura en el cultivo de marango, mientras en el cultivo de pitahaya con el mismo tipo de trampa la abundancia total fue de 2438 insectos.

Mairena (2015), reporta que en el cultivo de piña encontró una abundancia total de 10657 especímenes, mientras en el cultivo de pitahaya con el mismo tipo de trampa la abundancia total fue de 2,438 insectos.

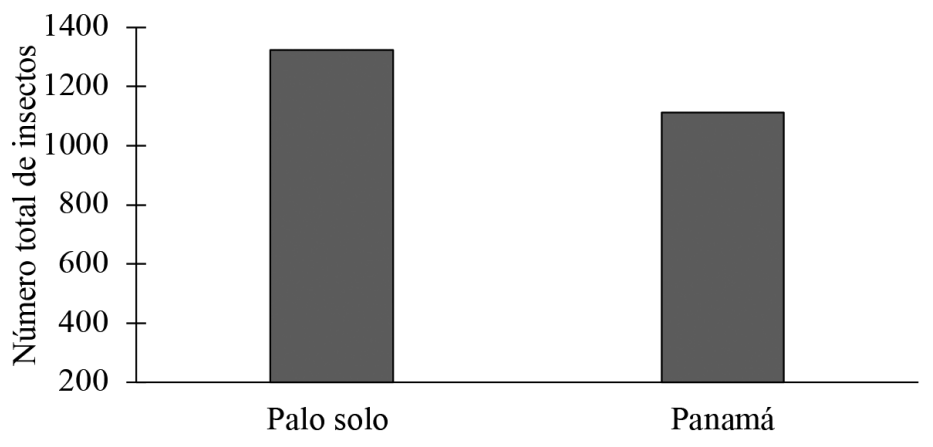

Fincas

Figura No. 1: Abundancia total de insectos encontrados en el cultivo de pitahaya 


\section{Riqueza total de familias de insectos encontrados en el cultivo de pitahaya}

En la Figura 2 se presenta la riqueza total de familias encontradas en el cultivo de pitahaya entre ambas fincas fue de 36 familias de insectos, siendo la finca Palo Solo, la que mayor riqueza de familias de insectos presentó, en cambió en la finca Panamá se capturaron 32 familias de insectos.

Lacayo y Mayorga (2014), reportan que en el cultivo de Marango (Moringa oleife$r a$ ), encontraron una riqueza de 15 familias de insectos, en trampas de caída libre y galones, mientras en el estudio de pitahaya en trampas de caída libre y galones fue de 36 familias de insectos. En el cultivo de marañón (Rugama y López, 2011), dicen que encontraron una riqueza total de 35 familias de insectos, en trampas de caída libre y galones, mientras en el estudio de pitahaya fue de 36 familias de insectos en el mismo tipo de trampas. Gómez, (2011), en el cultivo de marañón, encontró una riqueza total de 11 familias de insectos, en trampas de caída libre y galones, en cambio en el estudio de pitahaya fue de 36 familias de insectos en trampas de caída libre y galones.

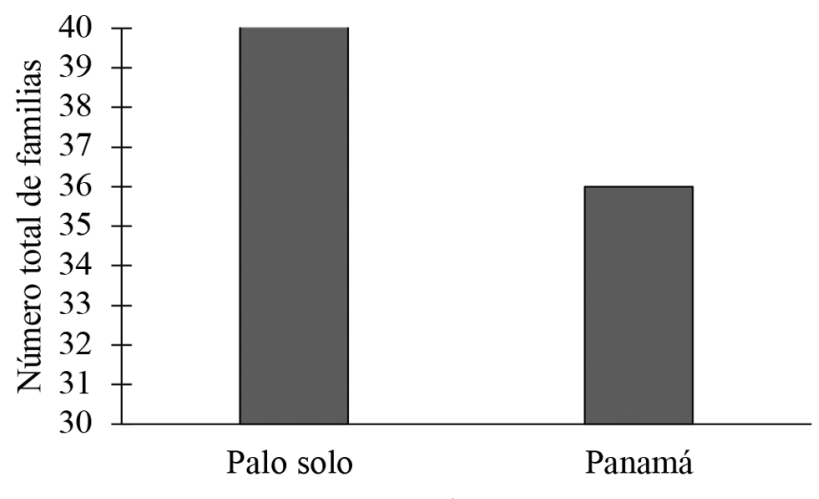

Finca

Figura No. 2: Riqueza total de familias de insectos encontrados en el cultivo de pitahaya.

\section{Distribución temporal de insectos de la familia Noctuidae en el cultivo de pitahaya}

Se encontró que las poblaciones de insectos de la familia Noctuidae se presentaron a partir de la fecha de colecta julio 15 hasta la última fecha de noviembre 12. Los mayores picos poblacionales de esta familia se presentaron en la finca Palo Solo, en las fechas del 15 de julio con 32 insectos, 30 de julio con 21 insectos y 28 octubre con 26 insectos, mientras en la finca Panamá los mayores picos poblacionales se presentaron el 14 de agosto con 31 insectos, 15 de julio con 18 insectos y el 30 de julio con 16 insectos. Los mayores picos poblacionales para esta familia, en las dos fincas fueron el 15 de julio, 14 de agosto y 28 de octubre (Figura 3). 
La familia Noctuidae pertenece al orden Lepidóptera, presenta metamorfosis completa (holometábola), son de tamaño pequeño a grande su extensión alar es de 15 a $140 \mathrm{~mm}$, cuerpo robusto, tapizado de escamas son generalmente de color gris o café, a veces las alas posteriores son de color crema a amarillo en la mayoría de los casos el ala anterior se presenta más delgada que la posterior (Nunes y Dávila, 2004), la mayoría de estos insectos vuelan de noche y son atraídos por la luz, también son atraídas por el azúcar y las flores ricas en néctar. Los adultos de la familia Noctuidae son considerados inofensivos, siendo el estado larval considerado de mucha importancia, debido a que son fitófagos, masticadores, barrenadores, cortadores, minadores, tejedores y algunos formadores de agallas, las larvas son del tipo eruciforme (Sáenz de la Llana, 1990. Jiménez, 2009).

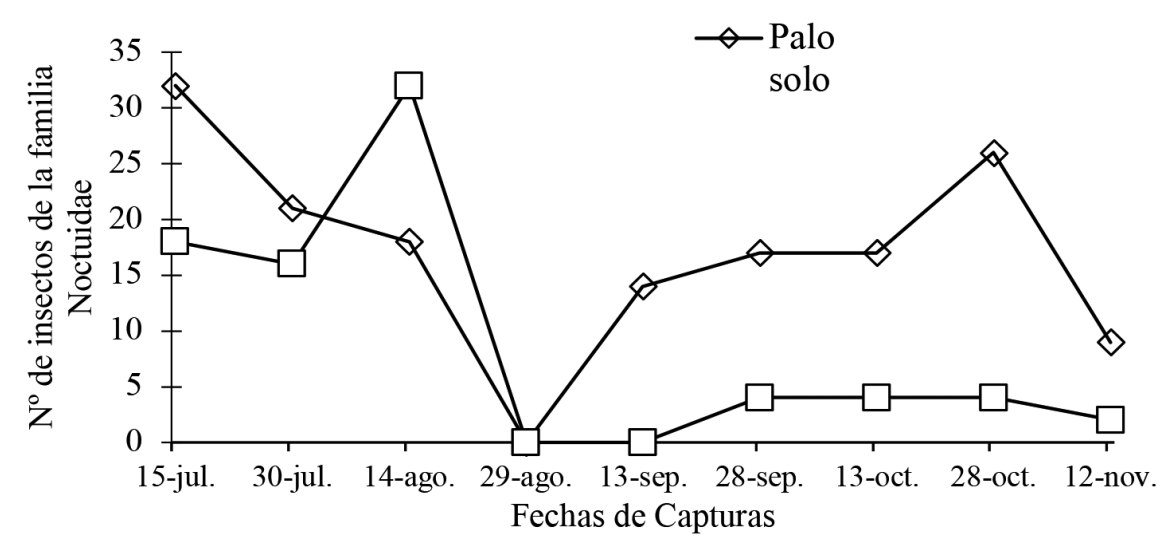

Figura No. 3: Distribución temporal de familias Noctuidae en el cultivo de pitahaya

\section{Distribución temporal de familia Chrysopidae en el cultivo de pitahaya}

Como resultado se muestra que hubo mayor población de insectos de esta familia en la finca Palo Solo con 9 de 9 fechas de colecta, en comparación con la finca Panamá donde se encontró 8 de 9 fechas de colectas. En los meses donde se presentaron los mayores picos poblacionales en la finca Palo Solo fue el 29 de agosto con 37 insectos, el 29 de agosto con 24 insectos y el 12 de noviembre 23 insectos, mientras que en la Panamá los mayores picos poblacionales fueron el 23 de septiembre con 23 insectos y el 2 de noviembre con 5 insectos colectados (Figura 4).

Según Santin (2017), la chrysopa en su estado larval este neuróptero juega un papel muy importante en el control biológico de la escama, ya que se alimenta de esta en sus estadios finales. Las larvas no son fácilmente identificables en el cladodio ya que se encuentran cubiertas por un polvillo blanco, semejante al que segregan las cochinillas, confundiéndose por lo tanto con estas y con las escamas de las cuales es un agente regulador. 


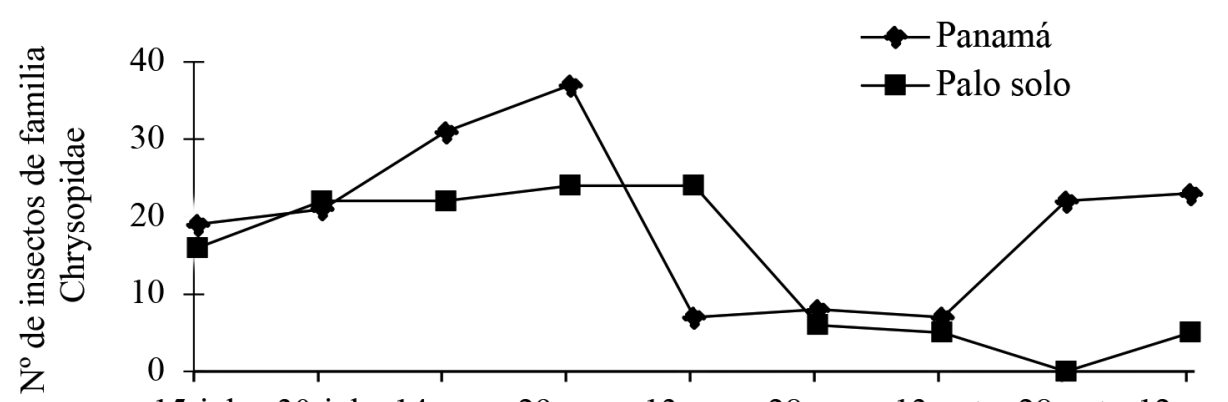

15-jul. 30-jul. 14-ago. 29-ago. 13-sep. 28-sep. 13-oct. 28-oct. 12-nov. Fechas de capturas

Figura No. 4: Distribución temporal de familias Chrysopidae en el cultivo de pitahaya.

\section{Distribución temporal de familias Phycitidae en el cultivo de pitahaya}

En la Figura 5 se muestran los resultados que se obtuvo mayor población de insectos de esta familia en la finca Palo Solo en las 9 fechas de colecta, en comparación con la finca Panamá donde no se encontró ningún insecto de esta familia en todas fechas de colectas. En los meses donde se presentaron los mayores picos poblacionales en la finca Palo Solo fue el 12 de noviembre con 53 insectos, el 13 de septiembre con 48 insectos y el 29 de agosto con 39 insectos, mientras que en la finca Panamá no se encontraron ningún insecto referente a la familia Phycitidae.

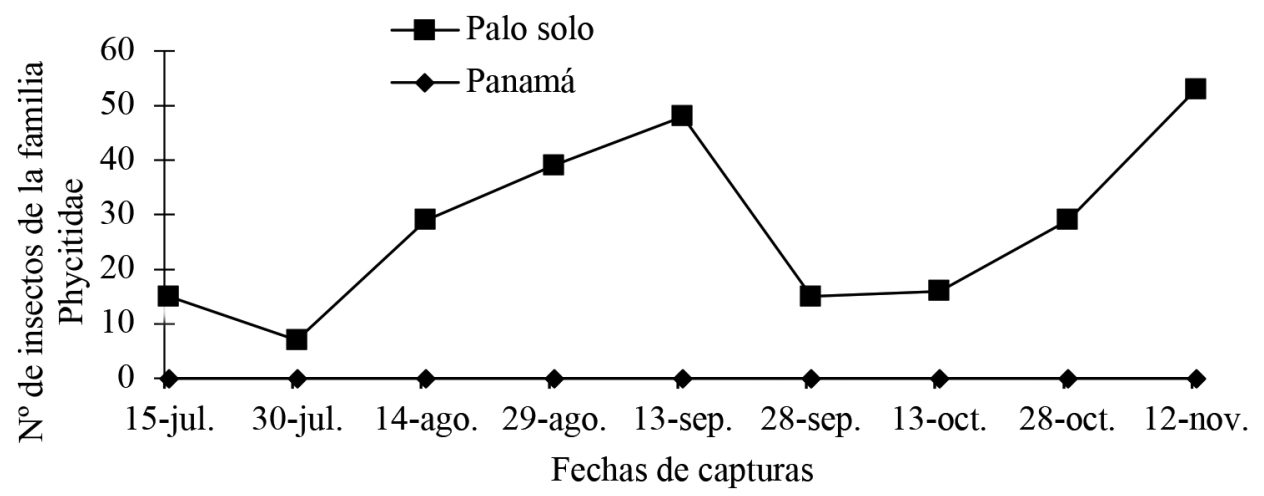

Figura No. 5: Distribución temporal de familias Phycitidae en el cultivo de pitahaya. 


\section{Conclusiones}

Se encontraron asociados a la pitahaya 9 órdenes y 40 familias de insectos, las familias más dominantes en la Finca Palo Solo fueron Phycitidae y Noctuidae y en la Finca Panamá fueron Chrysopidae y Hesperiidae.

La mayor abundancia, riqueza y diversidad de insectos asociados al cultivo de la pitahaya fue encontrada en la finca Palo Solo y los mayores picos poblacionales en su distribución temporal fueron encontrados en las familias Noctuidae y Chrysopidae. La mayor captura de insectos fue registrada en la trampa de caída libre en la finca Palo solo.

\section{Recomendaciones}

Se recomienda establecer los genotipos Orejona y Sabaneña por sus características morfológicas y la tolerancia a enfermedades.

Se recomienda asociar el cultivo de pitahaya con otros cultivos alimenticios para que la diversidad insectil aumente.

\section{Agradecimiento}

Esta publicación obtuvo el financiamiento de: El Fondo de Asistencia Internacional de los Estudiantes y Académicos Noruegos (SAIH).

\section{Lista de referencia}

Andrews Keith. L., y Caballero, R. (1989). Guía para el estudio de órdenes y familias de insectos de Centroamérica. Francisco Morazán, Honduras: Escuela Agrícola Panamericana El Zamorano.

Avelares Santos, J. J Fernández, M. V., Gómez, G. O., y Guido, M. A. (1996). Recolección de germoplasma de pitahayas (Hylocereus Undatus Britton \& Rose) efectuado en 13 departamentos de la zona del Pacifico y central de Nicaragua: En Memorias del segundo Encuentro Nacional sobre el cultivo de la pitahaya. Managua, Nicaragua.

Becerra, O. A. (1986). El Cultivo de la pitahaya. Bogotá, Colombia: Federación Nacional de Cafetaleros de Colombia.

Gómez Martínez. J. (2011). Entomofauna y patógenos asociados al cultivo de marañón (Anacardium occidentale L.), en León, Nicaragua, entre los meses de julio 2009 a marzo 2010. (Tesis de maestría). Recuperada de http://repositorio.una.edu. ni/2167/1/tnh1og633p.pdf 
Instituto Nicaragüense de Estudios Territoriales (INETER) (2008). Caracterización climática del departamento de Carazo. Recuperado de http://www.bvsde.org. ni/Web_textos/INETER/INETERoo63/5.\%20Caracterizacion\%2oclimatica\%20 Carazo.doc

Instituto Nicaragüense de Tecnología Agropecuaria. (2002). Guía tecnológica del cultivo de la pitahaya. Managua, Nicaragua: INTA.

Jiménez Martínez, E. S. (2009). Texto Básico: Entomología. Managua Nicaragua: UNA. 140 Pp.

Lacayo Rodríguez, R. T., y Mayorga Mendoza, J. R. (2014). Abundancia, riqueza y diversidad insectil asociada al cultivo de moringa (Moringa oleífera L.) en Managua, Nicaragua, Entre los meses de mayo a diciembre del 2013. (Tesis de grado). Recuperada de http://repositorio.una.edu.ni/3156/1/tnh1ol129a.pdf

Maes, J. M. (1998). Insectos de Nicaragua: catálogo de los insectos y artrópodos terrestres de Nicaragua. León, Nicaragua.

Mairena Vásquez, C. L. (2015). Identificación y fluctuación poblacional de insectos asociados al cultivo de la piña (Ananas comusus L. Merril.), en Ticuantepe, Nicaragua, Entre los meses de marzo a septiembre 2014. (Tesis de maestría). Recuperada de http://repositorio.una.edu.ni/3363/1/tnh1om228.pdf

Ministerio de Fomento Industria y Comercio. (2001). Norma Técnica Obligatoria Nicaragüense Norma de Procedimientos para la Producción, Comercialización y Exportación de la Fruta Fresca y Pulpa de Pitahaya. Managua, Nicaragua: MIFIC.

Montano Núñez, R. G. y Bustamante Maradiaga, E. J. (2017). Entomofauna, diversidad $y$ fluctuación poblacional de insectos plagas y benéficos asociados al cultivo de la maracuyá (Passiflora edulis Sims), en Sébaco, Matagalpa, 2016. (Tesis de grado). Recuperada de http://repositorio.una.edu.ni/3456/1/tnh1om765.pdf

Nunes Zuffo, C., y Dávila Arce, M. L. (2004). Taxonomía de las Principales Familias y Subfamilias de Insectos de interés Agrícolas en Nicaragua. Estelí, Nicaragua: UCATSE.

Rosses, L. M. (1999). Estudio sobre la caracterización, producción y rentabilidad del cultivo de la pitahaya. Managua, Nicaragua: MAG.

Rugama Lovo, I. M., y López Vílchez, M. E. (2011). Identificación y descripción de los principales insectos rastreros asociados al cultivo de marañón (Anacardium occidentale L.), orgánico y convencional en León, Nicaragua, durante los meses de agosto 
2009 a marzo 2010. (Tesis de grado) Recuperada de http://repositorio.una.edu. ni/2142/1/tnh1org28i.pdf

Sáenz, M., y De La Llana, A. (1990). Entomología sistemática. Managua, Nicaragua: UNA.

Santin J. (2017). Plagas y enfermedades de la tuna Opuntia ficus indica L. en las condiciones ecológicas de la provincia de Loja. Recuperado de : https://www.researchgate. net/publication/317954097_Plagas_y_enfermedades_de_la_tuna_Opuntia_ficus_indica_L_en_las_condiciones_ecologicas_de_la_provincia_de_Loja_Pests_ and_diseases_of_tuna_Opuntia_ficus_indica_L_In_the_ecological_conditions_of_the_prov

Téllez Manzanares, M. S., y Jirón Cortez, V. M. (2014). Identificación y variación poblacional de insectos asociados al cultivo de marango (Moringa oleífera L.) en Managua, Nicaragua durante los meses de noviembre 2012 a abril 2013. (Tesis de grado) Recuperada de http://repositorio.una.edu.ni/2756/1/tnh1ot275i.pdf

Universidad Nacional Agraria (UNA) (2008). Guías y Formas Metodológicas de la Forma de Culminación de estudios. Managua, Nicaragua: UNA. 